\title{
EXISTENCE OF SOLUTIONS FOR THE AW-RASCLE TRAFFIC FLOW MODEL WITH VACUUM
}

\author{
MARTE GODVIK AND HARALD HANCHE-OLSEN
}

\begin{abstract}
We consider the macroscopic model for traffic flow proposed by Aw and Rascle in 2000 . The model is a $2 \times 2$ system of hyperbolic conservation laws, or, when the model includes a relaxation term, a $2 \times 2$ system of hyperbolic balance laws. The main difficulty is the presence of vacuum, which makes us unable to control the total variation of the conservative variables. We allow vacuum to appear and prove existence of a weak entropy solution to the Cauchy problem.
\end{abstract}

\section{INTRODUCTION}

In [2] Aw and Rascle introduced a new macroscopic model for traffic flow,

$$
\left.\begin{array}{rl}
\rho_{t}+(\rho v)_{x} & =0 \\
(\rho w)_{t}+(\rho w v)_{x} & =0
\end{array}\right\} \quad \text { where } w=v+p(\rho),
$$

the functions $\rho(x, t)$ and $v(x, t)$ are the density and the velocity of cars on the roadway and $x \in \mathbb{R}$ and $t \in \mathbb{R}^{+}$are the Eulerian space and time variable, respectively. For simplicity we write the system as

$$
u_{t}+f(u)_{x}=0,
$$

where $u=(\rho, y)=(\rho, \rho w) \in \mathcal{U} \subset \mathbb{R}^{2}$. The function $p(\rho)$ is smooth and strictly increasing and it satisfies

$$
p(0)=0, \quad \lim _{\rho \rightarrow 0} \rho p^{\prime}(\rho)=0 \quad \text { and } \rho p^{\prime \prime}(\rho)+2 p^{\prime}(\rho)>0 \text { for } \rho>0 .
$$

The last assumption ensures strict hyperbolicity for $\rho>0$. The prototype of the function $p(\rho)$ is

$$
p(\rho) \propto \rho^{\gamma}, \quad \gamma>0 .
$$

The eigenvalues of the system are

$$
\lambda_{1}=v-\rho p^{\prime}(\rho) \text { and } \lambda_{2}=v .
$$

For $\rho>0$ the first wave family is genuinely nonlinear and the second family is linearly degenerate. Moreover, for $\rho=0$ the eigenvalues coincide and the system is only hyperbolic. In [2] Aw and Rascle solve the Riemann problem for this model and they include the vacuum state. For a discussion of the model, see also [1], [5], [6], [8], [9], [10], [11], [12] and [15].

The model is of Temple class, i.e., the shock and rarefaction curves coincide. The Riemann invariants are $w$ and $v$, and the wave curves are given by $w=$ const. and $v=$ const., respectively. A solution of the Riemann problem that does not include vacuum consists of at most two waves, one of each family. Thus, any subdomain $\mathcal{D} \subset \mathcal{U}$ defined by

$$
\mathcal{D}=\left\{u \in \mathcal{U}: w_{-} \leq w(u) \leq w_{+}, 0 \leq v_{-} \leq v(u) \leq v_{+}\right\},
$$

Date: June 12, 2007. 


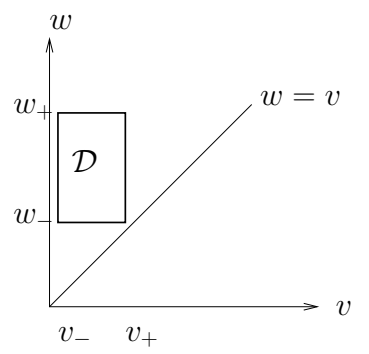

(a)

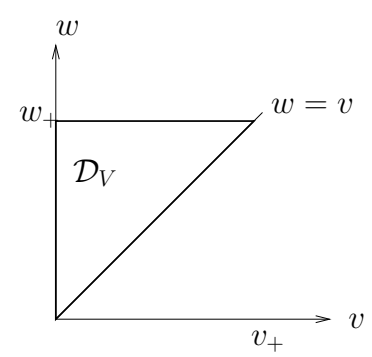

(b)

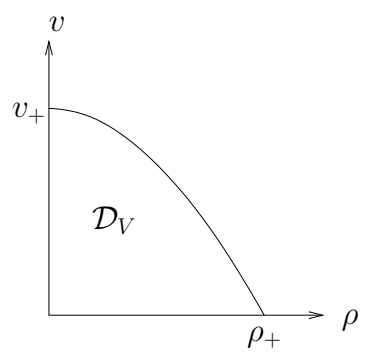

(c)

Figure 1. The domains (a) $\mathcal{D}$ and (b)-(c) $\mathcal{D}_{V}$.

where $w_{-}>v_{+}$, is invariant for the Riemann problem. Further, for a solution of the Riemann problem with initial data in $\mathcal{D}$, the total variation of the Riemann invariants is nonincreasing. The domain $\mathcal{D}$ is shown in figure $1(\mathrm{a})$. If we pick initial values $u_{0}(x)$ in $\mathcal{D}$, vacuum will not appear in the solution of the Cauchy problem. Thus the function $\left(p^{-1}\right)^{\prime}$ is bounded, and it is possible to obtain a bound on the total variation of $(\rho, y)$ from the total variation of the Riemann invariants. By using this property and the Glimm scheme [7], it can be shown that the Cauchy problem, with $u(x, 0)=u_{0}(x) \in \mathrm{BV}(\mathbb{R})^{2}$, has a weak entropy solution. This argument is given in Serre [16, Chapter 5].

As long as we must exclude the vacuum state, our choice of an invariant domain is severely limited. If we, for example, want to increase the maximal velocity $v_{+}$, we also have to increase $w_{-}$in order to stay away from vacuum. When including the vacuum state, the available invariant domains are given by (4) requiring $w(u) \geq$ $v(u)$ instead of $w_{-}>v_{+}$. All these regions are subdomains of

$$
\mathcal{D}_{V}=\left\{u \in \mathcal{U}: 0 \leq v(u) \leq w(u) \leq v_{+}\right\} .
$$

The domain $\mathcal{D}_{V}$ is depicted in figure $1(\mathrm{~b})-1(\mathrm{c})$. Note that we now can include any nonnegative value of the car density $\rho_{0}$ and velocity $v_{0}$ in $\mathcal{D}_{V}$ by choosing a larger value of $v_{+}$.

A Riemann problem with left state $u_{L}$ and right state $u_{R}$ produces a vacuum state at time $t=0^{+}$if and only if

$$
v_{R} \geq w_{L} .
$$

When the Riemann data satisfies this condition, the solution consists of a rarefaction wave that connects $u_{L}$ to a vacuum state given by $w=v=w_{L}$, and a contact wave that connects the vacuum state given by $v=w=v_{R}$ to the right state $u_{R}$. Hence the total variation of the Riemann invariants is still nonincreasing. So, if $p^{\prime}(\rho)>\epsilon>0$, it is in general possible to obtain a bound on the total variation of $(\rho, y)$ from the total variation of the Riemann invariants, and the Glimm scheme yields existence of a weak entropy solution.

In [19] it is shown for the $p$-system that, unless vacuum is present initially or appears immediately, the solution will not reach a vacuum state in finite time. This is not the case for the Aw-Rascle model. In order to show this, assume initial Cauchy data consisting of three constant states denoted by $u_{L}, u_{M}$ and $u_{R}$ such that $v_{L}=v_{M}, w_{M}=w_{R}$ and $w_{L} \leq v_{R}$. Further, assume that the Cauchy data does not include vacuum. Thus, at time $t=0^{+}$the solution consists of a contact discontinuity separating the leftmost state $u_{L}$ and the middle state $u_{M}$, and a rarefaction wave connecting $u_{M}$ and the rightmost state $u_{R}$. The solution, as a function of time and space, is shown in figure 2(a), and figure 2(b) gives the states in 


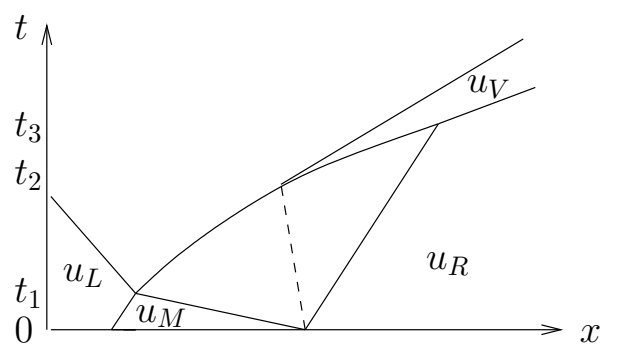

(a)

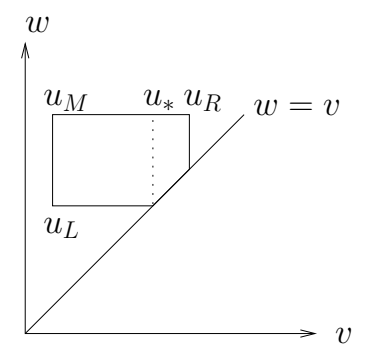

(b)

FiguRE 2. A contact discontinuity and a rarefaction wave collide and a vacuum state $u_{V}$ appears in the solution. The state along the dashed characteristic in figure (a) is the state denoted by $u_{*}$ in figure (b).

the $(w, v)$-plane. The contact travels faster than the rarefaction, and the two waves will collide at time $t_{1}$. Since $w_{L} \leq v_{R}$, the resultant state once the incoming waves have passed through each other includes vacuum. Across the incoming rarefaction wave the velocity $v$ increases. Thus, as the contact discontinuity transverses the rarefaction, the velocity of the contact increases. Since the velocity of the rightmost part of the incoming rarefaction is $\lambda_{1}\left(u_{R}\right)<\lambda_{2}\left(u_{R}\right)$, the contact travels through the rarefaction in finite time and leave the rarefaction at time $t=t_{3}$. Thus, vacuum will appear in the solution at some time $t_{2}$. Further, several waves can collide at the same time. Then vacuum appears if and only if (6) is satisfied for $L$ denoting the leftmost wave and $R$ the rightmost wave.

We want to show existence of a weak entropy solution of the Cauchy problem for system (1), with initial data $u_{0}(x)$ taking values in $\mathcal{D}_{V}$. Assume

$$
p^{\prime}(0)=0, \quad p^{\prime}(\rho)>0 \text { for } \rho>0, \quad \text { and }\left|p\left(\rho_{1}\right)-p\left(\rho_{2}\right)\right| \leq L\left|\rho_{1}-\rho_{2}\right|,
$$

for some constant $L$, which is satisfied for the prototype function given by (3) with $\gamma>1$. Since we allow vacuum states and $p^{\prime}(0)=0$, we are unable to control the total variation of the conservative variables, and thus we can not use the Glimm scheme to show existence. However, we consider slightly modified systems for which we can control the total variation and show existence of weak entropy solutions. The Riemann invariants have nonincreasing total variation and they are Lipschitz continuous in time as functions into $L_{l o c}^{1}(\mathbb{R})$. Thus, by introducing a cut-off function and using a compactness argument, we show convergence of a sequence of weak entropy solutions of the slightly modified systems. It turns out that the limit is a weak solution of the original system. However, when we include vacuum the system has no strictly convex entropy $\eta(\rho, y)$. We relax the assumptions on the entropy function and assume only that $\eta(\rho, y)$ is strictly convex in $\rho$. For this choice of entropy functions we show that admissible, in the sense of Lax, discontinuous solutions of the Riemann problem for (1) satisfy the entropy inequality, and that it fails for inadmissible discontinuities. Finally, we show that the weak solution obtained for the Cauchy problem for (1) satisfies the entropy inequality. In Section 2 we prove the following theorem:

Theorem 1. Let the initial Riemann invariants $\left(w_{0}(x), v_{0}(x)\right)$ be in $\mathrm{BV}(\mathbb{R})^{2}$ and take values in $\mathcal{D}_{V}$. Assume $p(\rho)$ satisfies (2) and (7). Then there exists a weak entropy solution $u(x, t)$ in $L_{\text {loc }}^{1}\left(\mathbb{R} \times \mathbb{R}^{+}\right)^{2}$ of system $(1)$ with initial data $u_{0}(x)$. Further, the total variation of the Riemann invariants $w(x, t)$ and $v(x, t)$ is nonincreasing. 
We expect our proof technique to work also for the network model [5], [12], provided that the junctions do not cause the total variation of $v$ and $w$ to blow up. It should also prove useful in overcoming any difficulty arising from loss of control of the total variation of $\rho$ near vacuum for related traffic models.

An improved version of the model (1) includes a relaxation term in the second equation,

$$
\begin{aligned}
& \rho_{t}+(\rho v)_{x}=0 \\
& y_{t}+(y v)_{x}=\rho R(\rho, y) .
\end{aligned}
$$

We assume the relaxation term satisfies

$$
R(\rho, y) \begin{cases}\geq 0 & \text { for } v=0, \quad w \leq w_{+}, \\ \leq 0, & \text { for } v \leq v_{+}, \quad w=w_{+}\end{cases}
$$

and is Lipschitz in $v$ and $w$,

$$
\left|R\left(\rho_{1}, y_{1}\right)-R\left(\rho_{2}, y_{2}\right)\right|<L\left(\left|v_{1}-v_{2}\right|+\left|w_{1}-w_{2}\right|\right) .
$$

By using operator splitting and Theorem 1 we obtain approximate solutions of the above system. The approximate Riemann invariants have bounded total variation and are Lipschitz continuous in time. Thus, by using the same technique as used to achieve Theorem 1, we prove, see Section 3, the following theorem:

Theorem 2. Assume the conditions of Theorem 1 are satisfied. Furthermore, assume $R(\rho, y)$ satisfies $(9)-(10)$. Then there exists a weak entropy solution $u(x, t)$ in $L_{l o c}^{1}\left(\mathbb{R} \times \mathbb{R}^{+}\right)^{2}$ of system (8) with initial data $u_{0}(x)$. Further, the total variation of the Riemann invariants $w(x, t)$ and $v(x, t)$ is bounded.

Remark. The operator splitting technique, as used in the proof of Theorem 2, also yields existence of a weak entropy solution of the Cauchy problem for strictly hyperbolic Temple systems with a source term. Consider the strictly hyperbolic Temple system $u_{t}+f(u)_{x}=g(u)$, where $u, f$ and $g$ are vectors in $\mathbb{R}^{n}$ and $(x, t) \in \mathbb{R} \times \mathbb{R}^{+}$. Assume the initial Riemann invariants, $r_{0}(x) \in \mathbb{R}^{n}$, have bounded total variation. We split the system into a homogenoues hyperbolic part, $u_{t}+f(u)_{x}=0$, and a system of ordinary differential equations, $u_{t}=g(u)$. Consider the homogeneous hyperbolic system. From the theory of Temple systems there exists a bounded invariant domain $\mathcal{D}$, and the Riemann invariants, $r \in \mathbb{R}^{n}$, have nonincreasing total variation and are Lipschitz continuous in time as function into $L_{l o c}^{1}(\mathbb{R})^{n}$. If the map $r \mapsto u(r)$ is a diffeomorphism, there exists a weak entropy solution of the Cauchy problem for the system. Consider the system of ordinary differential equations, and assume that the domain $\mathcal{D}$ is an invariant domain for the system. Denote $r=R(u)$ and $u=U(r)$. Thus, by a change of variables the system transforms to $r_{t}=\mathrm{d} R(U(r)) g(U(r))$. If $g, U$ and $\mathrm{d} R$ are Lipschitz, Grönwall's inequality yields $\mathrm{TV}(r(t)) \leq e^{C t} \mathrm{TV}\left(r_{0}\right)$, for a constant $C$, and Lipschitz continuity in time of $r$. Finally, the same operator splitting technique as used to prove Theorem 2, yields existence of a weak entropy solution $u(x, t)$ of the inhomogeneous hyperbolic system, and the solution has bounded total variation. In [4] Colombo and Corli prove well-posedness for a class of strictly hyperbolic Temple systems with a source, assuming the eigenvalues of the system are separated on every compact subset of $\mathcal{D}$. The above proof holds for more general systems, but we only obtain existence of a weak entropy solution. In particular, the eigenvalues of the Aw-Rascle model are not separated on $\mathcal{D}_{V}(5)$, so [4] is not directly applicable.

A specific choice of the relaxation term, as given in [15], is

$$
R(\rho, v)=\frac{1}{\tau}(V(\rho)-v)=\tilde{R}(w, v)=\frac{1}{\tau}\left(V \circ p^{-1}(w-v)-v\right),
$$




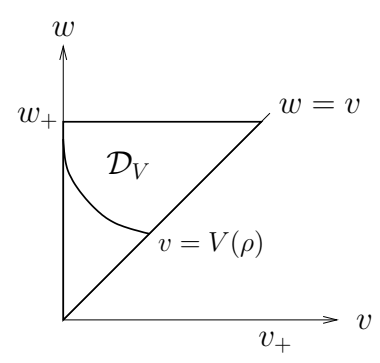

Figure 3. The curve $v=V(\rho)$ and the domain $\mathcal{D}_{V}$.

where the constant $\tau$ is the relaxation time and the smooth function $V(\rho)$ is an equilibrium velocity. Further, assume the subcharacteristic condition, see [15],

$$
-p^{\prime}(\rho) \leq V^{\prime}(\rho) \leq 0
$$

is satisfied.

We want to show that (9)-(10) are satisfied with this particular choice of $R(\rho, v)$ under these extra conditions: There is some density $\rho_{0}>0$ so that

$$
w_{+} \geq p\left(\rho_{0}\right) \quad \text { and } \quad V(\rho) \begin{cases}>0, & 0 \leq \rho<\rho_{0}, \\ =0, & \rho>\rho_{0} .\end{cases}
$$

The top half of (9) is trivially satisfied. As to the bottom half, note that (12) implies $\tilde{R}_{v} \leq 0$ and $w_{+} \geq p\left(\rho_{0}\right)$ implies $p^{-1}\left(w_{+}\right) \geq \rho_{0}$. Thus $\tilde{R}\left(v, w_{+}\right) \leq \tilde{R}\left(0, w_{+}\right)=$ $\tau^{-1} V \circ p^{-1}\left(w_{+}\right)=0$. The domain $\mathcal{D}_{V}$ and a curve $V(\rho)$ are shown in figure 3 .

The Lipschitz condition (10) follows too, since (12) implies $-\tau^{-1} \leq \tilde{R}_{v} \leq 0$ and $-\tau^{-1} \leq \tilde{R}_{w} \leq 0$, and (10) is satisfied with $L=\tau^{-1}$. In conclusion, if we assume (12)-(13), the assumptions in Theorem 2 hold for $R(\rho, v)$ given by (11). Thus there exists a weak entropy solution including vacuum for system (8) with this specific choice of relaxation term.

In [17] Siebel and Mauser introduce another source term $\rho R(\rho, v)$ to the second equation in the Aw-Rascle model. Their function $R(\rho, v)$ satisfies (9)-(10), and by Theorem 2 there exits a weak entropy solution of the system. The source term is motivated by experimental data and the system gives an unstable regime for intermediate densities. For the unstable region the equilibrium density curves in the fundamental diagram is shifted towards an inverse- $\lambda$ shape. This feature is observed in traffic dynamics, as discussed in [14]. Another approach for modeling this instability using the Aw-Rascle model is given in [8].

Before proving the theorems we introduce some notation. Let $\Omega$ denote any bounded subset of $\mathbb{R}$. The purpose is to compute in $L^{1}(\Omega)$ and tacitly draw conclusions about $L_{l o c}^{1}(\mathbb{R})$. The norm on $L^{1}(\Omega)$ is denoted by $\|\cdot\|$. Further, we will usually omit subscripts on subsequences and let any subsequence of the sequence $u^{\delta}$ be denoted by $u^{\delta}$. In particular, whenever speaking of convergence of $u^{\delta}$, we really mean convergence of some subsequence. For simplicity, we write $u(t)$ instead of $u(\cdot, t)$. Finally, let $a \vee b$ denote $\max (a, b)$.

\section{Proof of Theorem 1.}

In order to prove Theorem 1, we first define an appropriate approximation of (1). For $\delta>0$, consider

$$
\begin{aligned}
\rho_{t}+(\rho v)_{x} & =0 \\
{\left[\rho\left(v+p^{\delta}(\rho)\right)\right]_{t}+\left[\rho v\left(\rho+p^{\delta}(\rho)\right)\right]_{x} } & =0,
\end{aligned}
$$


where

$$
p^{\delta}(\rho)= \begin{cases}\frac{p(\delta)}{\delta} \rho, & \rho \leq \delta \\ p(\rho), & \rho>\delta,\end{cases}
$$

and $w=v+p^{\delta}(\rho)$. The initial Riemann invariants $v_{0}(x)$ and $w_{0}(x)$ are in $B V(\mathbb{R})$ and take values in $\mathcal{D}_{V}$. Further, we take them to be independent of $\delta$. The initial data $u_{0}^{\delta}(x)$ depends on $\delta$ since $w_{0}=v_{0}+p^{\delta}\left(\rho^{\delta}\right)$. Assume $\delta<\rho_{+}$, where $\rho_{+}=$ $\left(p^{\delta}\right)^{-1}\left(w_{+}\right)=p^{-1}\left(w_{+}\right)$is the maximal density. We denote the solution of the conservative problem by $\left(\rho^{\delta}, y^{\delta}\right)$ and the Riemann invariants by $\left(v^{\delta}, w^{\delta}\right)$. The modified system is also of Temple class. As discussed in the previous section, the total variation of the Riemann invariants, even when including the vacuum state, is nonincreasing,

$$
\mathrm{TV}\left(w^{\delta}(t), v^{\delta}(t)\right) \leq \mathrm{TV}\left(w_{0}, v_{0}\right) \leq C
$$

where $C$ is a constant. For $\delta>0$ the function $p^{\delta}(\rho)$ satisfies

$$
C_{\delta}\left|p^{\delta}\left(\rho_{1}\right)-p^{\delta}\left(\rho_{2}\right)\right| \geq\left|\rho_{1}-\rho_{2}\right|,
$$

where $C_{\delta}=\left\|1 /\left(p^{\delta}\right)^{\prime}\right\|_{\infty}$. From $p^{\delta}\left(\rho^{\delta}\right)=w^{\delta}-v^{\delta}$ we find

$$
\mathrm{TV}\left(\rho^{\delta}(t)\right) \leq C_{\delta} \operatorname{TV}\left(w^{\delta}(t), v^{\delta}(t)\right) \leq C_{\delta} C,
$$

which implies

$$
\begin{aligned}
\mathrm{TV}\left(y^{\delta}(t)\right) & \leq \rho_{+} \mathrm{TV}\left(w^{\delta}(t)\right)+w_{+} \mathrm{TV}\left(\rho^{\delta}(t)\right) \\
& \leq \rho_{+} C+w_{+} C_{\delta} C .
\end{aligned}
$$

Thus, for some constant $M_{\delta}$ depending on $\delta$, we have

$$
\operatorname{TV}\left(\rho^{\delta}(t), y^{\delta}(t)\right) \leq M_{\delta} .
$$

Notice that $M_{\delta}$ increases without bound as $\delta \rightarrow 0$, because the same is true for $C_{\delta}$.

For initial Riemann data taking values in $\mathcal{D}_{V}$, there exists a solution to the Riemann problem for (14)-(15) with $\delta>0$, see [2]. The domain $\mathcal{D}_{V}$ is an invariant region in the sense that if the initial data lies in $\mathcal{D}_{V}$, then so does the solution. Consider the Cauchy problem for (14)-(15) with initial Riemann invariants $\left(v_{0}(x), w_{0}(x)\right)$ in $B V(\mathbb{R})^{2}$ taking values in $\mathcal{D}_{V}$ and initial Cauchy data $u_{0}^{\delta}(x)$. Since $\mathcal{D}_{V}$ is bounded, the Glimm approximate solutions can be defined for all times $t$, and they are bounded. Further, the total variation of the Glimm approximations are bounded. Thus, by Serre [16, Theorem 5.4.1], the Glimm scheme yields existence of a weak entropy solution $u^{\delta}(x, t)$ of the Cauchy problem. The solution is bounded in $L^{\infty}(\mathbb{R} \times[0, T])^{2}$ and the total variation is given by (19). Further, the solution is Lipschitz continuous in time,

$$
\left\|u^{\delta}(t)-u^{\delta}(s)\right\| \leq 2 \mathrm{TV}\left(u^{\delta}(t)\right)|t-s| \leq M_{\delta}|t-s|,
$$

for $s \leq t \leq T$.

In order to show that the Riemann invariants are Lipschitz in time independently of $\delta$, we consider the Glimm scheme as given in [16, Chapter 5]. We denote by $u_{h}^{\delta}(t)$ and $\left(w_{h}^{\delta}(t), v_{h}^{\delta}(t)\right)$ the approximate solution and the approximate Riemann invariants of (14) given by the Glimm scheme at time $t$. Let $h=\Delta x=c \Delta t$, where $\Delta x$ and $\Delta t$ are the space and time step, respectively, and $c$ is some constant. For a fixed $t$ in $[0, T]$, it is shown that $u_{h}^{\delta}(t)$ converges to $u^{\delta}(t)$ in $L_{l o c}^{1}(\mathbb{R})^{2}$ as $h \rightarrow 0$. Further, since the Riemann invariants are bounded in $L^{\infty}(\mathbb{R})$ and have bounded total variation independently of $h$, Helly's theorem yields the existence of a subsequence converging to some limit $\left(w^{\delta}(t), v^{\delta}(t)\right)$ in $L_{l o c}^{1}(\mathbb{R})^{2}$. The limits should satisfy $w^{\delta}=v^{\delta}+p^{\delta}\left(\rho^{\delta}\right)$ and $y^{\delta}=\rho^{\delta} w^{\delta}$. Since $p^{\delta}$ is Lipschitz in its argument and 
$w_{h}^{\delta}=v_{h}^{\delta}+p^{\delta}\left(\rho_{h}^{\delta}\right)$, the first equality is satisfied. Further, since $y_{h}^{\delta}=\rho_{h}^{\delta} w_{h}^{\delta}$, the second equality is satisfied.

A diagonal argument gives us a subsequence $\left(w_{h}^{\delta}(t), v_{h}^{\delta}(t)\right)$ converging for all $t$ in $\mathcal{S}$, where $\mathcal{S}$ is a countable and dense subset of $[0, T]$. By the same technique as used in [16, Chapter 5.4] when proving that $u_{h}^{\delta}(t)$ is Lipschitz continuous in time, it can be shown, for any $s \leq t$ in $[0, T]$, that

$$
\begin{aligned}
\left\|w_{h}^{\delta}(t)-w_{h}^{\delta}(s)\right\|+\left\|v_{h}^{\delta}(t)-v_{h}^{\delta}(s)\right\| & \leq 2 \mathrm{TV}\left(w_{h}^{\delta}(t), v_{h}^{\delta}(t)\right)(|t-s|+h) \\
& \leq C(|t-s|+h) .
\end{aligned}
$$

Notice by (16) that the constant $C$ is independent of $\delta$. Assume $t \in[0, T]$ and $t \notin \mathcal{S}$. Let $t_{k}$ be a sequence in $\mathcal{S}$ such that $t_{k} \rightarrow t$ as $k \rightarrow \infty$. Finally,

$$
\begin{aligned}
\left\|v_{h_{m}}^{\delta}(t)-v_{h_{n}}^{\delta}(t)\right\| \leq\left\|v_{h_{m}}^{\delta}(t)-v_{h_{m}}^{\delta}\left(t_{k}\right)\right\|+\| v_{h_{m}}^{\delta}\left(t_{k}\right)- & v_{h_{n}}^{\delta}\left(t_{k}\right) \| \\
& +\left\|v_{h_{n}}^{\delta}\left(t_{k}\right)-v_{h_{n}}^{\delta}(t)\right\| .
\end{aligned}
$$

By the Lipschitz continuity of $v_{h}^{\delta}$ in time, the first and last terms can be made arbitrary small by choosing $k$ large. Fixing such a $k$, if $m$ and $n$ are large, the middle term is small. Thus, the sequence $v_{h}^{\delta}$ is Cauchy in $L_{l o c}^{1}\left(\mathbb{R} \times \mathbb{R}^{+}\right)$. Further,

$$
\left\|v^{\delta}(t)-v^{\delta}(s)\right\| \leq\left\|v^{\delta}(t)-v_{h}^{\delta}(t)\right\|+\left\|v_{h}^{\delta}(t)-v_{h}^{\delta}(s)\right\| \quad+\left\|v_{h}^{\delta}(s)-v^{\delta}(s)\right\| .
$$

Apply (20) to the middle term and let $h \rightarrow 0$. The same arguments hold for $w^{\delta}$ and therefore the Riemann invariants are Lipschitz in time,

$$
\left\|w^{\delta}(t)-w^{\delta}(s)\right\|+\left\|v^{\delta}(t)-v^{\delta}(s)\right\| \leq C|t-s|
$$

Since the Riemann invariants $w^{\delta}(t)$ and $v^{\delta}(t)$ are bounded in $L^{\infty}(\mathbb{R})$ and have bounded total variation independently of $\delta$, Helly's theorem yields the existence of a subsequence $\left(w^{\delta}, v^{\delta}\right)$ converging to some limit in $L_{l o c}^{1}(\mathbb{R})^{2}$. Using the same argument as for the convergence when $h \rightarrow 0$, we end the above discussion by the following conclusion:

Lemma 1. $\left(w^{\delta}, v^{\delta}\right)$ converges to some limit $(w, v)$ in $L_{l o c}^{1}\left(\mathbb{R} \times \mathbb{R}^{+}\right)^{2}$ as $\delta \rightarrow 0$ and the limit $(v, w)$ satisfies

$$
\mathrm{TV}(w(t), v(t)) \leq \mathrm{TV}\left(w_{0}, v_{0}\right) \leq C
$$

and

$$
\|w(t)-w(s)\|+\|v(t)-v(s)\| \leq C|t-s| .
$$

The above properties of the limit $(w, v)$ follows directly from (16) and (21).

We are going to prove convergence of a subsequence $u^{\delta}(t)$ to some limit in $L_{l o c}^{1}(\mathbb{R})$ by using the following result:

Lemma 2. Let $K$ be a set of nonnegative functions uniformly bounded in $L^{\infty}(\mathbb{R})$. If, for any $\epsilon>0$, there is a constant $M_{\epsilon}$ so that $\mathrm{TV}(u \vee \epsilon) \leq M_{\epsilon}$ for all $u \in K$, then the set $K$ is precompact in $L_{\text {loc }}^{1}(\mathbb{R})$.

Proof. For a fixed $\epsilon>0$, let $K_{\epsilon}=\{u \vee \epsilon: u \in K\}$. We have $u \vee \epsilon$ bounded in $L^{\infty}(\mathbb{R})$ and $\operatorname{TV}(u \vee \epsilon) \leq M_{\epsilon}$. Thus, by Helly's theorem, the set $K_{\epsilon}$ is precompact in $L_{\text {loc }}^{1}(\mathbb{R})$. Since $u$ is bounded in $L^{\infty}(\mathbb{R})$, we have $K \subset L_{\text {loc }}^{1}(\mathbb{R})$. Further, since $\|u-u \vee \epsilon\|<\epsilon|\Omega|$ for all $u \in K$, we find $\operatorname{dist}\left(u, K_{\epsilon}\right)<\epsilon|\Omega|$ for all $u$ in $K$. By [13, Lemma A.4], $K$ is precompact in $L_{\text {loc }}^{1}(\mathbb{R})$. 
Fix $t \in[0, T]$ and define the set $K=\left\{\rho^{\delta}(t)\right\}_{\delta>0}$. The solution $\rho^{\delta}(t)$ takes values in $\mathcal{D}_{V}$, and hence $K \subset L^{\infty}(\mathbb{R})$. Consider the total variation of $\rho^{\delta} \vee \epsilon$. Since

$$
\frac{1}{\left(p^{\delta}\right)^{\prime}(\rho \vee \epsilon)} \leq \begin{cases}1 / p^{\prime}(\epsilon), & \delta \leq \epsilon \\ 1 /\left(p^{\epsilon}\right)^{\prime}(\epsilon), & \delta>\epsilon,\end{cases}
$$

where $p^{\epsilon}$ is defined by (15) replacing $\delta$ by $\epsilon$, the constant $C_{\delta}$ given in inequality (17) now depends on $\epsilon$ instead of $\delta$. Thus, the same argument as used to obtain (18) yields

$$
\operatorname{TV}\left(\rho^{\delta} \vee \epsilon\right) \leq M_{\epsilon}, \quad \text { for all } \delta \geq 0 .
$$

By Lemma 2 the set $K$ is precompact in $L_{\text {loc }}^{1}(\mathbb{R})$. Thus $\rho^{\delta}(t)$ converges in $L_{\text {loc }}^{1}(\mathbb{R})$ to some limit as $\delta \rightarrow 0$. A diagonal argument gives us a subsequence $\rho^{\delta}(t)$ converging for all $t \in \mathcal{S}$.

Lemma 3. $\left(\rho^{\delta}, y^{\delta}\right)$ converges to some limit $(\rho, y)$ in $L_{l o c}^{1}\left(\mathbb{R} \times \mathbb{R}^{+}\right)$as $\delta \rightarrow 0$.

Proof. It remains to show convergence of $u^{\delta}(t)$ for all $t$ in $[0, T]$. Let

$$
\rho_{\epsilon}^{\delta}=\epsilon \vee \rho^{\delta}, \quad y_{\epsilon}^{\delta}=\left(\epsilon \vee \rho^{\delta}\right) w^{\delta}, \quad \text { for all } \epsilon>0 .
$$

Denote $u_{\epsilon}^{\delta}=\left(\rho_{\epsilon}^{\delta}, y_{\epsilon}^{\delta}\right)$. Notice that $u_{\epsilon}^{\delta}$ converges uniformly to $u^{\delta}$ as $\epsilon \rightarrow 0$. From the previous paragraph we have $\rho_{\epsilon}^{\delta}(t)$ converging to some limit $\rho_{\epsilon}(t)$ for all $t$ in $\mathcal{S}$. Then, $y_{\epsilon}^{\delta} \rightarrow y_{\epsilon}=\rho_{\epsilon} w$, as the factors converge in $L_{l o c}^{1}$ and are uniformly bounded in $L^{\infty}$. Hence we can conclude that the sequence $u_{\epsilon}^{\delta}(t)$ converges to some limit $u_{\epsilon}(t)$ in $L_{\text {loc }}^{1}(\mathbb{R})^{2}$ for all $t \in \mathcal{S}$. The Lipschitz continuity in time of $w^{\delta}$ and $v^{\delta}$ yields Lipschitz continuity in time for $p^{\delta}\left(\rho^{\delta}\right)=w^{\delta}-v^{\delta}$,

$$
\left\|p^{\delta}\left(\rho^{\delta}(t)\right)-p^{\delta}\left(\rho^{\delta}(s)\right)\right\| \leq C|t-s| .
$$

By using $(17),(24)$ and the above inequality we can show that $\rho_{\epsilon}^{\delta}(t)$ is Lipschitz continuous in time for all $t, s$ in $[0, T]$,

$$
\begin{aligned}
\left\|\rho_{\epsilon}^{\delta}(t)-\rho_{\epsilon}^{\delta}(s)\right\| & \leq\left\|\frac{1}{\left(p^{\delta}\right)^{\prime}\left(\rho_{\epsilon}^{\delta}\right)}\right\|_{\infty}\left\|p^{\delta}\left(\rho_{\epsilon}^{\delta}(t)\right)-p^{\delta}\left(\rho_{\epsilon}^{\delta}(s)\right)\right\| \\
& \leq C_{\epsilon}|t-s|,
\end{aligned}
$$

where the constant $C_{\epsilon}$ depends on $\epsilon$. Also, $y_{\epsilon}^{\delta}(t)$ is Lipschitz continuous in time,

$$
\begin{aligned}
\left\|y_{\epsilon}^{\delta}(t)-y_{\epsilon}^{\delta}(s)\right\| & \leq\left\|\rho_{\epsilon}^{\delta}(t)\left(w^{\delta}(t)-w^{\delta}(s)\right)\right\|+\left\|\left(\rho^{\delta}(t)-\rho_{\epsilon}^{\delta}(s)\right) w^{\delta}(s)\right\| \\
& \leq\left(\rho_{+} C+w_{+} C_{\epsilon}\right)|t-s| .
\end{aligned}
$$

Now, assume $t \in[0, T]$ and $t \notin \mathcal{S}$ and let $t_{k}$ be a sequence in $\mathcal{S}$ such that $t_{k} \rightarrow t$ as $k \rightarrow \infty$. Consider

$$
\begin{aligned}
\left\|u_{\epsilon}^{\delta_{m}}(t)-u_{\epsilon}^{\delta_{n}}(t)\right\| \leq\left\|u_{\epsilon}^{\delta_{m}}(t)-u_{\epsilon}^{\delta_{m}}\left(t_{k}\right)\right\|+\| u_{\epsilon}^{\delta_{m}}\left(t_{k}\right) & -u_{\epsilon}^{\delta_{n}}\left(t_{k}\right) \| \\
& +\left\|u_{\epsilon}^{\delta_{n}}\left(t_{k}\right)-u_{\epsilon}^{\delta_{n}}(t)\right\| .
\end{aligned}
$$

Since $u_{\epsilon}^{\delta}$ is Lipschitz continuous in time with a Lipschitz constant independent of $\delta$, the first and third term can be made small by choosing $k$ large, i.e. the term $\left|t-t_{k}\right|$ is small. For a fixed $k$, the middle term is small for $m$ and $n$ large. Hence the sequence is Cauchy in $L_{l o c}^{1}(\mathbb{R})^{2}$, and $u_{\epsilon}^{\delta}(t)$ converges to $u_{\epsilon}(t)$ for all $t \in[0, T]$. Finally, consider the sequence $u^{\delta}(t)$ and some $t$ in $[0, T]$. We have

$$
\begin{aligned}
\left\|u^{\delta_{m}}(t)-u^{\delta_{n}}(t)\right\| \leq\left\|u^{\delta_{m}}(t)-u_{\epsilon}^{\delta_{m}}(t)\right\|+\| u_{\epsilon}^{\delta_{m}}(t)- & u_{\epsilon}^{\delta_{n}}(t) \| \\
& +\left\|u_{\epsilon}^{\delta_{n}}(t)-u^{\delta_{n}}(t)\right\| .
\end{aligned}
$$

Since $\epsilon$ is arbitrary, the first and third term can be made small by choosing $\epsilon$ small. Then, by choosing $m$ and $n$ large, the middle term is small. Hence the sequence 
is Cauchy in $L_{l o c}^{1}(\mathbb{R})^{2}$, and there exists a sequence $u^{\delta}(t)$ converging to some limit $u(t)$ in $L_{l o c}^{1}(\mathbb{R})^{2}$ as $\delta \rightarrow 0$.

The function $u^{\delta}(x, t)$ is a weak solution of the approximate problem (14) with initial data $u_{0}^{\delta}(x)$. Thus, for all test functions $\phi(x, t) \in C_{0}^{\infty}\left(\mathbb{R} \times \mathbb{R}^{+}\right)$,

$$
\int_{\mathbb{R}} \int_{\mathbb{R}^{+}}\left(u^{\delta} \phi_{t}+f^{\delta}\left(u^{\delta}\right) \phi_{x}\right) \mathrm{d} t \mathrm{~d} x+\int_{\mathbb{R}} u_{0}^{\delta} \phi(x, 0) \mathrm{d} x=0
$$

where

$$
f^{\delta}(u)=\left(y-\rho p^{\delta}(\rho), y\left(w-p^{\delta}(\rho)\right)\right) .
$$

The function $f^{\delta}$ converges uniformly to $f$. Since $u^{\delta}$ and $\left(w^{\delta}, v^{\delta}\right)$ are bounded, $f^{\delta}\left(u^{\delta}\right)$ converges in $L_{l o c}^{1}$ to $f(u)$ as $\delta \rightarrow 0$. Thus Lebesgue's dominated convergence theorem yields

$$
\int_{\mathbb{R}} \int_{\mathbb{R}^{+}}\left(u \phi_{t}+f(u) \phi_{x}\right) \mathrm{d} t \mathrm{~d} x+\int_{\mathbb{R}} u_{0}(x) \phi(x, 0) \mathrm{d} x=0 .
$$

For all $t \in[0, T]$, the weak solution $u^{\delta}(t)$ of system $(14)$ converges in $L_{l o c}^{1}(\mathbb{R})$ to a weak solution $u(t)$ of system (1). Further, the convergence is in $C\left([0, T] ; L_{l o c}^{1}(\mathbb{R})^{2}\right)$. The limits satisfy $w=v+p(\rho)$ and $y=\rho w$ and thus $w(x, t)$ and $v(x, t)$ are the Riemann invariants of system (1).

It remains to prove that the limit $u(x, t)$ is a weak entropy solution of (1) with initial data $u_{0}$. An entropy/entropy flux pair $(\eta, q)$ for a hyperbolic system is an entropy $\eta: \mathcal{U} \rightarrow \mathbb{R}$ and a flux $q: \mathcal{U} \rightarrow \mathbb{R}$ satisfying

$$
\nabla_{u} q=\nabla_{u} \eta \mathrm{d} f
$$

where $\mathrm{d} f$ is the Jacobian matrix of $f(u)$. By [16] we know that $u^{\delta}$ is a weak entropy solution of (14),

$$
\int_{\mathbb{R}} \int_{\mathbb{R}^{+}}\left(\eta^{\delta}\left(u^{\delta}\right) \phi_{t}+q^{\delta}\left(u^{\delta}\right) \phi_{x}\right) \mathrm{d} t \mathrm{~d} x+\int_{\mathbb{R}} \eta^{\delta}\left(u_{0}^{\delta}\right) \phi(x, 0) \mathrm{d} x \geq 0,
$$

for all nonnegative test functions $\phi(x, t) \in C_{0}^{\infty}(\mathbb{R} \times \mathbb{R})$ and all convex entropies $\eta^{\delta}$ with corresponding flux $q^{\delta}$. Consider system (1). Using the transformation given by Wagner [18, Theorem 1],

$$
\frac{\partial X}{\partial x}=\rho, \quad \frac{\partial y}{\partial t}=-\rho v
$$

where $X(x, t)$ is the Lagrangian mass coordinate, we rewrite system (1) as

$$
\begin{aligned}
\tau_{t}+(\tilde{p}(\tau)-w)_{X} & =0 \\
w_{t} & =0
\end{aligned}
$$

where $\tau=1 / \rho$ and $\tilde{p}(\tau)=p(\rho)$. For $0<\rho \leq \rho_{+}$, system (1) is equivalent to the above system and the relation between the entropy/entropy flux pairs $(\eta, q)$, in Eulerian coordinates, and $(\tilde{\eta}, \tilde{q})$, in Lagrangian coordinates, is

$$
\begin{aligned}
& \tilde{\eta}(\tau, w)=\frac{1}{\rho} \eta(\rho, y) \\
& \tilde{q}(\tau, w)=q(\rho, y)-\rho v \tilde{\eta}(\tau, w) .
\end{aligned}
$$

Moreover, the entropy $\eta$ is convex if and only if $\tilde{\eta}$ is convex.

Any entropy/entropy flux pair $(\eta, q)$ of $(33)$ must satisfy

$$
\begin{aligned}
\tilde{q}_{\tau}-\tilde{p}^{\prime}(\tau) \tilde{\eta}_{\tau} & =0 \\
\tilde{\eta}_{\tau}+\tilde{q}_{w} & =0 .
\end{aligned}
$$


Solving this system yields

$$
\begin{aligned}
& \tilde{q}(\tau, w)=g(v), \\
& \tilde{\eta}(\tau, w)=h(w)-\int_{\tau_{0}}^{\tau} g^{\prime}(w-\tilde{p}(\xi)) \mathrm{d} \xi,
\end{aligned}
$$

where $\tau_{0}$ is a constant value and $h=h(w)$ and $g=g(v)$ are smooth functions in $L^{\infty}(\mathbb{R})$ having continuous second and third order derivatives, respectively. In order to obtain strict convexity of $\eta$ we compute

$$
\begin{aligned}
\tilde{\eta}_{\tau \tau} & =g^{\prime \prime}(v) \tilde{p}^{\prime}(\tau), \\
\tilde{\eta}_{\tau w} & =\tilde{\eta}_{w \tau}=-g^{\prime \prime}(v), \\
\tilde{\eta}_{w w} & =h^{\prime \prime}(w)-\int_{\tau_{0}}^{\tau} g^{\prime \prime \prime}(w-\tilde{p}(\xi)) \mathrm{d} \xi .
\end{aligned}
$$

The Hessian matrix of $\tilde{\eta}$ is positive definite if $\tilde{\eta}_{\tau \tau}>0$ and $\tilde{\eta}_{\tau \tau} \tilde{\eta}_{w w}>\left(\tilde{\eta}_{\tau w}\right)^{2}$. Since $(\tilde{p})^{\prime}<0$, we require $g^{\prime \prime}<0$ and

$$
\tilde{p}^{\prime}(\tau)\left(h^{\prime \prime}(w)-\int_{\tau_{0}}^{\tau} g^{\prime \prime \prime}(w-\tilde{p}(\xi)) \mathrm{d} \xi\right)<g^{\prime \prime}(v) .
$$

For fixed $\tau$ and $w$, the above inequality is satisfied for $h^{\prime \prime}$ big enough. However, when $w$ is fixed and $\tau \rightarrow \infty$ the left hand side of inequality (35) is asymptotically equal to $-\tilde{p}^{\prime}(\tau) \tau g^{\prime \prime \prime}(w)$, which goes to 0 as $\tau \rightarrow \infty$. Thus we have to require $g^{\prime \prime}=0$, and we conclude that there are no strictly convex entropies when vacuum is included.

We now define a semiconvex entropy $\eta$ with corresponding flux $q$ as a an entropy satisfying $\eta_{\rho \rho}>0$ for $\rho>0$. Further, an entropy solution is a weak solution which satisfies an entropy inequality for all such entropy/entropy flux pairs. The relation between $\eta$ and $\tilde{\eta}$ yields $\eta_{\rho \rho}=\tau^{3} \tilde{\eta}_{\tau \tau}$. Thus, for $\rho>0$ we have $\eta_{\rho \rho}>0$ if and only if $\tilde{\eta}_{\tau \tau}>0$. Since $h(w)_{t}=h^{\prime}(w) w_{t}=0$ in Lagrangian coordinates and we now have a weaker assumption on $\eta$, we can, for simplicity, choose $h(w)=0$. Thus the semiconvex entropy/entropy flux pairs of system (33) are

$$
\tilde{q}(\tau, w)=g(w-\tilde{p}(\tau)), \quad \tilde{\eta}(\tau, w)=-\int_{\tau_{0}}^{\tau} g^{\prime}(w-\tilde{p}(\xi)) \mathrm{d} \xi,
$$

where $g(v)$ is a smooth function such that $g^{\prime \prime}(v)<0$. A calculation of the above entropy/entropy flux pairs is also done in [3].

Lemma 4. The admissible discontinuities satisfy the entropy inequality for all semiconvex entropies with corresponding entropy fluxes and the entropy inequality fails for the inadmissible discontinuities.

Proof. Discontinuous solutions $u$ satisfy the Rankine-Hugionot condition,

$$
s\left(u_{R}-u_{L}\right)=f\left(u_{R}\right)-f\left(u_{L}\right),
$$

where $s$ is the speed of the discontinuity and $L$ and $R$ denote the left and right state, respectively. Further, by Lax' admissibility condition a shock of the first family is admissible if

$$
\lambda_{1}\left(u_{R}\right) \leq s \leq \lambda_{1}\left(u_{L}\right) .
$$

Consider the Riemann problem for system (33) in Lagrangian coordinates. The eigenvalues are

$$
\tilde{\lambda}_{1}=\tilde{p}^{\prime}(\tau) \text { and } \tilde{\lambda}_{2}=0 .
$$

For $\tau<\infty$ the first family is genuinely nonlinear and the second family is linearly degenerate. Moreover, at the vacuum state $\tilde{\lambda}_{1}=\tilde{\lambda}_{2}=0$. The wave curves are given by $w=$ const. and $v=$ const., respectively. Then, consider a point on an isolated 
discontinuity and a test function $\phi(x, t)$ whose support lies entirely inside a small neighborhood of this point. By Green's theorem an entropy inequality as given in (32) is satisfied for $\tilde{u}$ if

$$
s\left(\tilde{\eta}\left(\tilde{u}_{R}\right)-\eta\left(\tilde{u}_{L}\right)\right) \geq \tilde{q}\left(\tilde{u}_{R}\right)-\tilde{q}\left(\tilde{u}_{L}\right) .
$$

Across contact discontinuities of the second wave family the velocity $v$ is preserved and $s$ equals 0 . Thus, for contact discontinuities the above inequality is satisfied with equality. Consider admissible shock solutions. Since $w$ is constant across a shock, both $\tau$ and $v$ should decrease across the jump, i.e. $v_{L}>v_{R}$ and $\tau_{L}>\tau_{R}$. In order to show that the admissible shocks do satisfy the entropy inequality, we insert the expressions of $\tilde{\eta}$ and $\tilde{q}$ into the above inequality, with the result,

$$
s \int_{\tau_{R}}^{\tau_{L}} g^{\prime}(w-\tilde{p}(\xi)) \mathrm{d} \xi \geq g\left(v_{R}\right)-g\left(v_{L}\right),
$$

where $w=w_{L}=w_{R}$. Further, inserting the expressions of $s$ given by the RankineHugionot condition into the above inequality yields

$$
\frac{1}{\tau_{L}-\tau_{R}} \int_{\tau_{R}}^{\tau_{L}} g^{\prime}(w-\tilde{p}(\xi)) \mathrm{d} \xi \begin{cases}\leq \frac{g\left(v_{R}\right)-g\left(v_{L}\right)}{v_{R}-v_{L}}, & v_{L}>v_{R}, \\ \geq \frac{g\left(v_{R}\right)-g\left(v_{L}\right)}{v_{R}-v_{L}}, & v_{L}<v_{R},\end{cases}
$$

where $w=w_{L}=w_{R}$. Consider the left hand side in the above inequality. By the substitution $\xi=t \tau_{L}+(1-t) \tau_{R}$ and using the strict convexity of $\tilde{p}$ and the fact that $g^{\prime}$ is decreasing, we get

$$
\begin{aligned}
\frac{1}{\tau_{L}-\tau_{R}} \int_{\tau_{R}}^{\tau_{L}} g^{\prime}(w-\tilde{p}(\xi)) \mathrm{d} \xi & =\int_{0}^{1} g^{\prime}\left(w-\tilde{p}\left(t \tau_{L}+(1-t) \tau_{R}\right)\right) \mathrm{d} t \\
& <\int_{0}^{1} g^{\prime}\left(w-t \tilde{p}\left(\tau_{L}\right)+(1-t) \tilde{p}\left(\tau_{R}\right)\right) \mathrm{d} t \\
& =\int_{0}^{1} g^{\prime}\left(t v_{L}+(1-t) v_{R}\right) \mathrm{d} t \\
& =\frac{g\left(v_{R}\right)-g\left(v_{L}\right)}{v_{R}-v_{L}} .
\end{aligned}
$$

Thus, the admissible shocks will satisfy, and the inadmissible shocks will violate, the entropy inequality (32) for all semiconvex entropies $\tilde{\eta}$ and corresponding entropy fluxes $\tilde{q}$.

By the previous lemma the solution of the Riemann problem for (14) satisfies (32) for all semiconvex entropy/entropy flux pairs $\left(\eta^{\delta}, q^{\delta}\right)$, and then so does the solution $u^{\delta}(x, t)$ of the Cauchy problem, see [16, Chapter 5.4].

By equation (34) and (36) the entropy/entropy flux pair $\left(\eta^{\delta}, q^{\delta}\right)$ of system (14) for $\rho>0$ is

$$
\begin{aligned}
\eta^{\delta}(\rho, y) & =\frac{1}{\tau} \tilde{\eta}^{\delta}(\tau, w)=\frac{1}{\tau} \int_{\tau_{0}}^{\tau} g^{\prime}\left(w-\tilde{p}^{\delta}(\xi)\right) \mathrm{d} \xi \\
q^{\delta}(\rho, y) & =\tilde{q}^{\delta}(\tau, w)+\frac{w-\tilde{p}^{\delta}(\tau)}{\tau} \tilde{\eta}^{\delta}(\tau, w) \\
& =g\left(w-\tilde{p}^{\delta}(\tau)\right)-\left(w-\tilde{p}^{\delta}(\tau)\right) \frac{1}{\tau} \int_{\tau_{0}}^{\tau} g^{\prime}\left(w-\tilde{p}^{\delta}(\xi)\right) \mathrm{d} \xi .
\end{aligned}
$$

Thus, $\eta^{\delta}(\rho, y) \rightarrow g^{\prime}(w)$ and $q^{\delta}(\rho, y) \rightarrow g(w)-w g^{\prime}(w)$ as $\rho \rightarrow 0$. The Lipschitz continuity of $g(v)$ and $g^{\prime}(v)$ with respect to $v$ yields uniform convergence of $\left(\eta^{\delta}, q^{\delta}\right)$ 
to some limit $(\eta, q)$ as $\delta \rightarrow 0$. Further, since $\mathrm{d} f^{\delta} \rightarrow \mathrm{d} f$, the limit $(\eta, q)$ satisfies $\nabla q=\mathrm{d} f \cdot \nabla \eta$.

Consider inequality (32). Let $\delta \rightarrow 0$ and use Lebesgue's dominated convergence theorem and the Lipschitz properties. Thus, we finally conclude that for all nonnegative test functions $\phi(x, t)$ in $C_{0}^{\infty}\left(\mathbb{R} \times \mathbb{R}^{+}\right)$and all semiconvex entropies $\eta$ with corresponding entropy fluxes $q$,

$$
\int_{\mathbb{R}} \int_{\mathbb{R}^{+}}\left(\eta(u) \phi_{t}+q(u) \phi_{x}\right) \mathrm{d} t \mathrm{~d} x+\int_{\mathbb{R}} \eta\left(u_{0}\right) \phi(x, 0) \mathrm{d} x \geq 0,
$$

and $u(x, t)$ is a weak entropy solution of (1).

\section{Proof of Theorem 2.}

Consider system (8) with initial Riemann invariants $\left(v_{0}(x), w_{0}(x)\right)$ in $B V(\mathbb{R})^{2}$ taking values in $\mathcal{D}_{V}$ and initial Cauchy data $u_{0}(x)$. In order to show existence of a weak entropy solution, we split the system into a hyperbolic part, given by (1), and a pair of ordinary differential equations in time, $\rho_{t}=0$ and $[\rho(v+p(\rho))]_{t}=\rho R(\rho, v)$. More conveniently we write

$$
\begin{aligned}
\rho_{t} & =0 \\
w_{t} & =v_{t}=R(\rho, v),
\end{aligned}
$$

where $v_{t}=w_{t}$ follows from $\rho(x, t)$ being constant in time. The hyperbolic system is treated in the previous section. For initial data given by $u_{0}(x)$, we denote a weak entropy solution of the hyperbolic part at time $t$ as $H(t) u_{0}(x)$. The domain $\mathcal{D}_{V}$ is an invariant domain for the system. Further, the total variation of the Riemann invariants is nonincreasing and they are Lipschitz continuous in time as functions into $L_{l o c}^{1}(\mathbb{R})$. Moreover, by Theorem 1 there exists a weak entropy solution in $L_{l o c}^{1}\left(\mathbb{R} \times \mathbb{R}^{+}\right)^{2}$.

Consider the system of ordinary differential equations (37) with initial data $u_{0}(x)$. Denote the solution at time $t$ by $S(t) u_{0}(x)$. For simplicity, we abuse this notation and write the variables $w, v$ and $y$ at time $t$ as $S(t) w_{0}(x), S(t) v_{0}(x)$ and $S(t) y_{0}(x)$, respectively. However, bear in mind that $S(t) w_{0}$ in fact depends on $v_{0}$ as well as $w_{0}$, and similarly for the other variables.

First, we want to show that if the initial data lies in the domain $\mathcal{D}_{V}$ given by (5), then so does the solution $S(t) u_{0}(x)$. Consider the boundary of the domain $\mathcal{D}_{V}$. When $w=w_{+}$we want $w_{t} \leq 0$, and thus we require $R(\rho, y) \leq 0$. On the part of the boundary where $v=0$ we want $v_{t} \geq 0$, which is satisfied if $R(\rho, y) \geq 0$. For $w=v$ we have $S(t) w=S(t) v$, and the solution is still the vacuum state. Thus, requiring (9) yields invariance of $\mathcal{D}_{V}$ with respect to the system of ordinary differential equations (37).

We now want to consider the total variation of the solution. From equation (37) we have

$$
\left(S(t) v_{1}-S(t) v_{2}\right)_{t}=R\left(S(t) \rho_{1}, S(t) v_{1}\right)-R\left(S(t) \rho_{2}, S(t) v_{2}\right) .
$$

Multiplying the equality by $\operatorname{sign}\left(S(t) v_{1}-S(t) v_{2}\right)$ yields

$$
\begin{aligned}
\left|S(t) v_{1}-S(t) v_{2}\right|_{t} & \leq\left|R\left(S(t) \rho_{1}, S(t) v_{1}\right)-R\left(S(t) \rho_{2}, S(t) v_{2}\right)\right| \\
& \leq L\left(\left|S(t) w_{1}-S(t) w_{2}\right|+\left|S(t) v_{1}-S(t) v_{2}\right|\right) .
\end{aligned}
$$

The same argument is true for $S(t) w$. Thus, by Grönwall's inequality,

$$
\left|S(t) w_{1}-S(t) w_{2}\right|+\left|S(t) v_{1}-S(t) v_{2}\right| \leq e^{L t}\left(\left|w_{1}-w_{2}\right|+\left|v_{1}-v_{2}\right|\right),
$$

and this implies

$$
\operatorname{TV}(S(t) w, S(t) v) \leq e^{L t} \operatorname{TV}(w, v) .
$$


Since $R(\rho, v)$ is bounded, $S(t) w$ and $S(t) v$ are Lipschitz continuous in time,

$$
\int_{\Omega}|S(t) w-S(s) w| \mathrm{d} x+\int_{\Omega}|S(t) v-S(s) v| \mathrm{d} x \leq \tilde{C}|t-s|,
$$

where $\tilde{C}=2\|R\|_{\infty}|\Omega|$.

We define an approximate solution of system (8) at time $t_{n}=n \Delta t$, where $\Delta t=$ $T / N$, by

$$
u^{n}=[H(\Delta t) S(\Delta t)]^{n} u_{0}(x) .
$$

Since $\mathcal{D}_{V}$ is invariant for both the hyperbolic part and the system of differential equations, the approximate solution $u^{n}$ takes values in $\mathcal{D}_{V}$. Further, by (22), (38) and induction the total variation of $w^{n}$ and $v^{n}$ is bounded,

$$
\mathrm{TV}\left(w^{n}, v^{n}\right) \leq C e^{L T} .
$$

By (23) and (39) we obtain Lipschitz continuity in time for $v^{n}$,

$$
\begin{aligned}
\left\|v^{n}-v^{n+m}\right\| & \leq \sum_{i=n}^{m-1}\left\|v^{i}-v^{i+1}\right\| \\
& \leq \sum_{i=n}^{m-1}\left(\left\|v^{i}-S(\Delta t) v^{i}\right\|+\left\|S(\Delta t) v^{i}-H(\Delta t) S(\Delta t) v^{i}\right\|\right) \\
& \leq \sum_{i=n}^{m-1}\left(\tilde{C}+C e^{L T}\right) \Delta t=\hat{C}\left|t_{n}-t_{n+m}\right| .
\end{aligned}
$$

The same argument holds for $w^{n}$.

We now consider an approximate solution that is defined for all times $t$ in $[0, T]$,

$$
u_{\Delta t}(x, t)= \begin{cases}H\left(2\left(t-t_{n}\right)\right) u^{n}(x), & t \in\left[t_{n}, t_{n+1 / 2}\right) \\ S\left(2\left(t-t_{n+1 / 2}\right)\right) u^{n+1 / 2}(x), & t \in\left[t_{n+1 / 2}, t_{n+1}\right),\end{cases}
$$

where $u^{n+1 / 2}(x)=H(\Delta t) u^{n}$. From the above results, $u_{\Delta t}$ takes values in the invariant domain $\mathcal{D}_{V}$. Further, $w_{\Delta t}$ and $v_{\Delta t}$ have bounded total variation,

$$
\mathrm{TV}\left(w_{\Delta t}, v_{\Delta t}\right) \leq C e^{L T},
$$

and they are Lipschitz continuous in time for $t_{n}, n$ in $\mathbb{N}$. By (23), (39) and (40), $w_{\Delta t}$ and $v_{\Delta t}$ are Lipschitz continuous in time,

$$
\left\|w_{\Delta t}(s)-w_{\Delta t}(t)\right\|+\left\|v_{\Delta t}(s)-v_{\Delta t}(t)\right\| \leq \bar{C}|s-t|,
$$

for all $s$ and $t$ in $[0, T]$ and a constant $\bar{C}$ independent of $\Delta t$.

In order to prove convergence of a subsequence $u_{\Delta}(x, t)$ to some limit in $L_{l o c}^{1}(\mathbb{R} \times$ $\mathbb{R}^{+}$), we use Lemma 2 and the same technique as we used to prove convergence of $u^{\delta}(x, t)$ in the previous section. Since $p^{\prime}\left(\rho_{\Delta t} \vee \epsilon\right)$ is bounded, equation (41) and $w_{\Delta t}=v_{\Delta t}+p\left(\rho_{\Delta t}\right)$ give

$$
\mathrm{TV}\left(\rho_{\Delta t} \vee \epsilon\right) \leq M_{\epsilon} \quad \text { for all } \Delta t \geq 0
$$

Thus, replacing $u^{\delta}$ by $u_{\Delta t}$ in equation (24)-(25) and (26)-(31) yields convergence of $u_{\Delta t}(x, t)$ to some limit $u(x, t)$ in $L_{l o c}^{1}\left(\mathbb{R} \times \mathbb{R}^{+}\right)^{2}$ as $\Delta t \rightarrow 0$. Further, the limits $w$ and $v$ also have bounded total variation (41) and they are Lipschitz continuous in time (42).

It remains to show that the limit $u(x, t)$ is a weak solution of system (8). For simplicity, introduce the vector $r(u)=(0, R(\rho, v))$. Since $u_{\Delta t}(x, t)$ is a weak solution of the hyperbolic system for $t \in\left[t_{n}, t_{n+1 / 2}\right)$, we have for all test functions 


$$
\begin{aligned}
& \phi(x, t) \in C_{0}^{\infty}\left(\mathbb{R} \times \mathbb{R}^{+}\right), \\
& \int_{\mathbb{R}} \int_{t_{n}}^{t_{n+1 / 2}}\left(u_{\Delta t} \phi_{t}+2 f\left(u_{\Delta t}\right) \phi_{x}\right) \mathrm{d} t \mathrm{~d} x \\
& \quad-\int_{\mathbb{R}} u_{\Delta t}\left(x, t_{n+1 / 2}\right) \phi\left(x, t_{n+1 / 2}\right) \mathrm{d} x+\int_{\mathbb{R}} u_{\Delta t}\left(x, t_{n}\right) \phi\left(x, t_{n}\right) \mathrm{d} x=0 .
\end{aligned}
$$

Further, $u_{\Delta t}(x, t)$ is a solution of the system of ordinary differential equations for $t \in\left[t_{n+1 / 2}, t_{n+1}\right)$. After multiplying with a test function $\phi$ and partial integration,

$$
\begin{aligned}
\int_{\mathbb{R}} \int_{t_{n+1 / 2}}^{t_{n+1}} u_{\Delta t} \phi_{t} \mathrm{~d} t \mathrm{~d} x+\int_{\mathbb{R}} \int_{t_{n+1 / 2}}^{t_{n+1}} 2 r\left(u_{\Delta t}\right) \phi \mathrm{d} t \mathrm{~d} x \\
-\int_{\mathbb{R}} u_{\Delta t}\left(x, t_{n+1}\right) \phi\left(x, t_{n+1}\right) \mathrm{d} x+\int_{\mathbb{R}} u_{\Delta t}\left(x, t_{n+1 / 2}\right) \phi\left(x, t_{n+1 / 2}\right) \mathrm{d} x=0 .
\end{aligned}
$$

Adding the two equations and summing over $n=0,1, \ldots, N-1$ yields

$$
\begin{gathered}
\int_{\mathbb{R}} \int_{0}^{T} u_{\Delta t} \phi_{t} \mathrm{~d} x \mathrm{~d} t+2 \chi_{\Delta t} \int_{\mathbb{R}} \int_{0}^{T} f\left(u_{\Delta t}\right) \phi_{x} \mathrm{~d} t \mathrm{~d} x \\
+2 \tilde{\chi}_{\Delta t} \int_{\mathbb{R}} \int_{0}^{T} r\left(u_{\Delta t}\right) \phi \mathrm{d} t \mathrm{~d} x+\int_{\mathbb{R}} u_{\Delta t}\left(x, t_{0}\right) \phi\left(x, t_{0}\right) \mathrm{d} x \\
\quad-\int_{\mathbb{R}} u_{\Delta t}(x, T) \phi(x, T) \mathrm{d} x=0,
\end{gathered}
$$

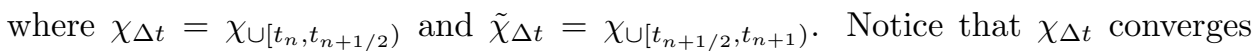
weakly to $\frac{1}{2}$ as $\Delta t \rightarrow 0$. Remember that $u_{\Delta t}$ is bounded and Lipschitz continuous in time. Thus, letting $\Delta t \rightarrow 0$ in the above equation, Lebesgue's dominated convergence theorem yields

$$
\begin{aligned}
\int_{\mathbb{R}} \int_{0}^{T}\left(u(x, t) \phi_{t}(x, t)+f\left(u(x, t) \phi_{x}\right) \mathrm{d} t\right. & \mathrm{d} x+\int_{\mathbb{R}} u_{0}(x) \phi(x, 0) \mathrm{d} x \\
& -\int_{\mathbb{R}} \int_{0}^{T} r(u(x, t)) \mathrm{d} t \phi(x, t) \mathrm{d} x=0
\end{aligned}
$$

i.e. the limit $u(x, t)$ is a weak solution of system (8).

In the proof of Theorem 1 we show existence of semiconvex entropy/entropy flux pairs $(\eta, q)$ of the flux $f$, where we assume that $\eta_{\rho \rho}>0$. By definition, $u_{\Delta t}$ is a weak entropy solution of the hyperbolic part for $t$ in $\left[t_{n}, t_{n+1 / 2}\right)$. Further, we multiply system (37) with $\nabla_{u} \eta \phi$ and integrate over $t$ in $\left[t_{n+1 / 2}, t_{n+1}\right)$. Thus, the same arguments as used to achieve (43) yields

$$
\begin{aligned}
\int_{\mathbb{R}} \int_{0}^{T}\left(\eta(u) \phi_{t}+q(u) \phi_{x}\right) \mathrm{d} x \mathrm{~d} t+\int_{\mathbb{R}} u_{0}(x) \phi(x, 0) \mathrm{d} x & \\
& +\int_{\mathbb{R}} \int_{0}^{T} \nabla_{u} \eta(u) r(u) \phi \mathrm{d} t \mathrm{~d} x \geq 0 .
\end{aligned}
$$

For all $t \in[0, T], u_{\Delta t}(t)$ converges in $L_{l o c}^{1}(\mathbb{R})^{2}$ to a weak entropy solution $u(t)$ of system $(8)$ and the convergence is in $C\left([0, T] ; L_{l o c}^{1}(\mathbb{R})^{2}\right)$. Further, the limits $w(x, t)$ and $v(x, t)$ are the Riemann invariants of system $(8)$.

Acknowledgments. The authors greatfully acknowledge the hospitality of the Mittag-Leffler Institute, Sweden (fall 2005, Nonlinear Waves program). The authors also acknowledge Denis Serre for valuable discussions during the stay at the Mittag-Leffler Institute. 


\section{REFERENCES}

[1] A. Aw, A. Klar, T. Materne, and M. Rascle. Derivation of continuum traffic flow models from microscopic follow-the-leader models. SIAM J. Appl. Math., 63(1):259-278 (electronic), 2002.

[2] A. Aw and M. Rascle. Resurrection of "second order" models of traffic flow. SIAM J.Appl. Math., 60(3):916-938 (electronic), 2000.

[3] P. Bagnerini and M. Rascle. A multiclass homogenized hyperbolic model of traffic flow. SIAM J. Math. Anal., 35(4):949-973 (electronic), 2003.

[4] R. M. Colombo and A. Corli. On a class of hyperbolic balance laws. J. Hyperbolic Differ. Equ., 1(4):725-745, 2004.

[5] M. Garavello and B. Piccoli. Traffic flow on a road network using the Aw-Rascle model. Comm. Partial Differential Equations, 31(1-3):243-275, 2006.

[6] M. Garavello and B. Piccoli. Traffic Flow on Networks. AIMS on Applied Math. American Series of Mathematical Sciences, 2006.

[7] J. Glimm. Solutions in the large for nonlinear hyperbolic systems of equations. Comm. Pure Appl. Math., 18:697-715, 1965.

[8] P. Goatin. The Aw-Rascle vehicular traffic flow model with phase transitions. Math. Comput. Modelling, 44(3-4):287-303, 2006.

[9] J. M. Greenberg. Extensions and amplifications of a traffic model of Aw and Rascle. SIAM J. Appl. Math., 62(3):729-745 (electronic), 2001/02.

[10] J. M. Greenberg. Congestion redux. SIAM J. Appl. Math., 64(4):1175-1185 (electronic), 2004.

[11] J. M. Greenberg, A. Klar, and M. Rascle. Congestion on multilane highways. SIAM J. Appl. Math., 63(3):818-833 (electronic), 2003.

[12] M. Herty and M. Rascle. Coupling conditions for a class of second-order models for traffic flow. SIAM J. Math. Anal., 38(2):595-616 (electronic), 2006.

[13] H. Holden and N. H. Risebro. Front tracking for hyperbolic conservation laws, volume 152 of Applied Mathematical Sciences. Springer-Verlag, New York, 2002.

[14] B. S. Kerner and H. Rehborn. Experimental Properties of Phase Transitions in Traffic Flow. Physical Review Letters, 79(20):4030-4033, 1997.

[15] M. Rascle. An improved macroscopic model of traffic flow: derivation and links with the Lighthill-Whitham model. Math. Comput. Modelling, 35(5-6):581-590, 2002. Traffic flowmodelling and simulation.

[16] D. Serre. Systems of conservation laws. 1. Cambridge University Press, Cambridge, 1999. Hyperbolicity, entropies, shock waves, Translated from the 1996 French original by I. N. Sneddon.

[17] F. Siebel and W. Mauser. On the fundamental diagram of traffic flow. SIAM J. Appl. Math., 66(4):1150-1162 (electronic), 2006.

[18] D. H. Wagner. Equivalence of the Euler and Lagrangian equations of gas dynamics for weak solutions. J. Differential Equations, 68(1):118-136, 1987.

[19] R. Young. The $p$-system. II. The vacuum. In Evolution equations (Warsaw, 2001), volume 60 of Banach Center Publ., pages 237-252. Polish Acad. Sci., Warsaw, 2003. 